\title{
When pressure meets light: detecting the photoacoustic effect at the origin
}

\author{
Junjie Yao \\ Light: Science \& Applications (2017) 6, e17062; doi:10.1038/Isa.2017.62; published online 2 June 2017
}

Modern optical microscopy ${ }^{1}$, including confocal microscopy, twophoton microscopy and optical coherence tomography (OCT), has revolutionized life sciences by providing detailed information of biological samples with cellular and subcellular resolutions, and has become an essential tool for biomedical research labs. However, optical microscopy typically has a limited penetration depth of $\sim 1 \mathrm{~mm}$ in biological tissue due to strong optical scattering ${ }^{2,3}$. Moreover, with respective contrast mechanisms, confocal and two-photon microscopy usually rely on fluorescent labeling of the samples and OCT still lacks sensitivity to many biological functions. In contrast, optical-resolution photoacoustic microscopy (OR-PAM) has emerged over the last decade as a complementary imaging tool to the existing optical microscopy by taking advantage of its unique optical absorption contrast ${ }^{4}$. By acoustically detecting the optical absorption in the tissue, OR-PAM has been proven a powerful tool for anatomical, functional and molecular imaging with endogenous or exogenous contrast agents. In particular, using hemoglobin as the endogenous optical absorber, OR-PAM currently represents the most sensitive blood detector and has been widely used for in vivo imaging of the blood perfusion and oxygenation, especially for cancer and brain studies. Nevertheless, the acoustic detection in OR-PAM is a doubleedged sword; on the one hand, it provides a relatively deep penetration with one-way optical attenuation and negligible acoustic attenuation, but on the other hand, the acoustic detection typically needs a coupling medium, such as water and ultrasound gel, between the tissue surface and the ultrasound transducer. The need for acoustic coupling has become one of the major factors that has hindered the wide adoption of OR-PAM by biomedical researchers whenever the biological samples are not compatible with an aquatic environment. Therefore, contact-free detection of the photoacoustic signals (that is, without the need for acoustic coupling) has captured the attention of the photoacoustic imaging community and resulted in many exciting advancements. If successful, the contact-free photoacoustic technologies will free up the working space and greatly expand the territory of PAM applications.

As optical detection of acoustic waves in ultrasound imaging, optical interferometry is the most popular approach in contact-free $\mathrm{PAM}^{5}$. Briefly, the detection light beam and the reference light beam are split from the same light source and then mixed. The mixed signal is perturbed by a photoacoustically generated thermal and pressure rise, which is then detected either locally (for example, via an OCT-based method) or at the sample's surface (for example, via a displacementbased method). However, the interferometry-based methods often suffer from the rather complex signal generation progress that is sensitive to phase noise and artifact induced by the light source, the target and the acoustic propagation environment, and thus have met only limited success in well-controlled lab settings.

To address the above issues, Hajireza et al. ${ }^{6}$ have reported a novel contact-free OR-PAM technology that is (1) free of a coupling medium for acoustic propagation, (2) free of interferometry that is vulnerable to phase noise, (3) maximally sensitive to the local photoacoustic pressure rise and (4) extremely easy to implement and highly compatible with other optical imaging modalities. The reported remote-sensing PAM (PARS) uses a low-coherence probe light beam (coherence length: $\sim 40 \mu \mathrm{m}$ ) to detect the local initial pressure rise (pressure magnitude: $\sim 100 \mathrm{MPa}$ ) following pulsed light excitation (pulse energy: $\sim 1 \mathrm{~nJ}$; Figure 1 ). The local pressure rise can substantially magnify the existing difference in refractive indices of the local optical absorber and the surrounding environment and thus change the reflectance of the probe beam. The change in the probe beam reflection can then be remotely recorded and is proportional to the original optical energy deposition inside the imaged sample.

Compared with the previous OR-PAM technologies ${ }^{7}$, Hajireza's work has a striking advantage: A substantially simplified imaging system. The all-optical excitation and detection configuration avoids the complex optical-acoustic combiners that are typically bulky and lossy and has paved the way for seamless combination of OR-PAM with other pure optical imaging modalities with complementary contrast mechanisms. Moreover, the detected optical signals are truly wideband and thus do not suffer from acoustic attenuation along the pathway. This advantage, however, is contaminated by a major drawback that acoustic flight-time cannot be recorded by the noninterferometry method and thus multiple targets at different depths will be mixed at the detector. In other words, the inherent acoustically determined depth resolution, as exists in traditional OR-PAM, is lost. 


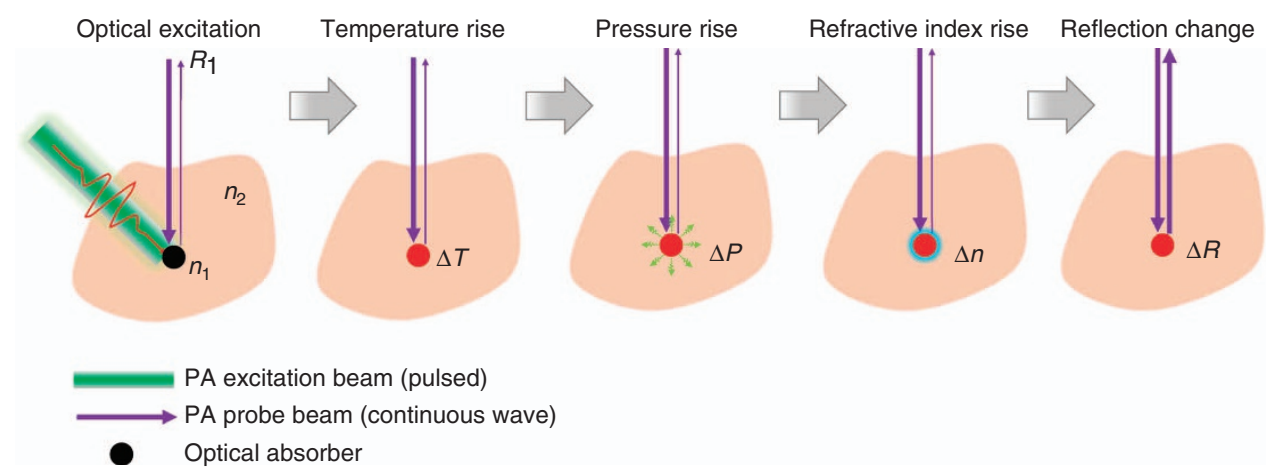

Figure 1 Remote-sensing photoacoustic microscopy. Hajireza et al., have developed a contact-free OR-PAM system that has achieved remote detection of local pressure rise by using a low-coherence probe light beam. Upon excitation by a pulsed laser light, the temperature of the local optical absorber increases, inducing a local pressure rise that changes the absorber's refractive index and the reflection of the probe beam.

This drawback could potentially be resolved by depth-gated or coherence-gated detection, at the cost of imaging speed or sensitivity.

The remote-sensing PAM technology has without a doubt opened a new window into many exciting technology breakthroughs and corresponding biomedical applications. (1) Reflection-mode ORPAM with a high-NA objective $(>1.0)$ is now possible, providing a lateral resolution of $\sim 200 \mathrm{~nm}$. The high-NA objective typically has a working distance of less than a few millimeters, so the all-optical detection configuration is well suited for the reflection-mode system. Super-resolution PAM can then be developed on top of the high-NA system. (2) Biological applications that are not water friendly can now be performed without damaging the samples (for example, C. elegans or Drosophila) or complicating the accompanying procedures (for example, intraoperative delineation of tumor margin). Moreover, midIR wavelengths that are strongly attenuated by water can now be used, allowing their corresponding endogenous absorbers such as water, lipids and glucose to be better imaged.

\section{CONFLICT OF INTEREST}

The author declares no conflict of interest.
1 Conchello JA, Lichtman JW. Optical sectioning microscopy. Nat Methods 2005; 2: 920-931.

2 Ntziachristos V. Going deeper than microscopy: the optical imaging frontier in biology. Nat Methods 2010; 7: 603-614.

3 Ntziachristos V, Ripoll J, Wang LV, Weissleder R. Looking and listening to light: the evolution of whole-body photonic imaging. Nat Biotechnol 2005; 23: 313-320.

4 Wang LV, Yao JJ. A practical guide to photoacoustic tomography in the life sciences. Nat Methods 2016; 13: 627-638.

5 Dong BQ, Sun C, Zhang HF. Optical detection of ultrasound in photoacoustic imaging. IEEE Trans Biomed Eng 2017; 64: 4-15.

6 Hajireza P, Shi W, Bell K, Paproski RJ, Zemp RJ. Non-interferometric photoacoustic remote sensing microscopy. Light Sci App/ 2017; 6: e16278.

7 Yao JJ, Wang LV. Photoacoustic microscopy. Laser Photonics Rev 2013; 7: 758-778.

(1)(2) This work is licensed under a Creative Commons Attribution(CY NC SA NonCommercial-ShareAlike 4.0 International License. The images or other third party material in this article are included in the article's Creative Commons license, unless indicated otherwise in the credit line; if the material is not included under the Creative Commons license, users will need to obtain permission from the license holder to reproduce the material. To view a copy of this license, visit http:// creativecommons.org/licenses/by-nc-sa/4.0/

(C) The Author(s) 2017 